\title{
Synthesis of SAPO-18 with low acidic strength and its application in conversion of dimethylether to olefins
}

Yuichiro Hirota $^{\mathrm{a}, *}$, Mayuka Yamada ${ }^{\mathrm{a}}$, Yoshiaki Uchida ${ }^{\mathrm{a}, \mathrm{b}}$, Yasuhiro Sakamoto ${ }^{\mathrm{b}, \mathrm{c}}$, Toshiyuki Yokoi $^{\mathrm{d}}$ and Norikazu Nishiyama ${ }^{a}$

${ }^{a}$ Division of Chemical Engineering, Graduate School of Engineering Science, Osaka University, 1-3 Machikaneyama, Toyonaka, Osaka 560-8531, Japan

${ }^{\mathrm{b}}$ PRESTO, Japan Science and Technology Agency, 4-1-8 Honcho, Kawaguchi, Saitama 332-0012, Japan

${ }^{\mathrm{c}}$ Department of Physics, Graduate School of Science, Osaka University, 1-1 Machikaneyama, Toyonaka, Osaka 560-8531, Japan

${ }^{\mathrm{d} C h e m i c a l ~ R e s o u r c e s ~ L a b o r a t o r y, ~ T o k y o ~ I n s t i t u t e ~ o f ~ T e c h n o l o g y, ~} 4259$ Nagatsuta, Midori-ku, Yokohama, Kanagawa 226-8503, Japan

*E-mail yhirota@cheng.es.osaka-u.ac.jp, Phone/FAX +81-6-6850-6257

\begin{abstract}
SAPO-18 molecular sieve was synthesized by a dry gel conversion (DGC) and hydrothermal (HT) synthesis method using methyltriethoxysilane as the only Si source (DGC-Me-SAPO-18 and HT-Me-SAPO-18). Their physicochemical and catalytic properties on dimethylether-to-olefin (DTO) reaction were compared with those of conventional SAPO-18 molecular sieves synthesized by a hydrothermal synthesis method (HT-SAPO-18). An $\mathrm{NH}_{3}$-TPD measurement revealed that the desorption peak position of Brønsted acid of the Me-SAPO-18 samples moved to lower temperature, indicating that Brønsted acidic strength of the Me-SAPO-18 samples was lower than that of the HT-SAPO-18 samples. The DME conversions and yields of light olefins over DGC-Me-SAPO-18 decreased at a slower rate than those of HT-SAPO-18. The decreased acidic strength and flake-like morphology of DGC-Me-SAPO-18 could retard the coke formation, leading to the prolonged catalytic lifetime.
\end{abstract}

Keywords: SAPO-18, dimethylether-to-olefin (DTO) reaction, dry gel conversion, Brønsted acid strength

\section{Introduction}

Availability of huge quantities of methane has led to an increased interest in catalytic methanol-to-olefin (MTO) and dimethylether-to-olefin (DTO) reactions, which are alternative routes to produce light olefins for subsequent polymerization and other chemical processes. Zeolite ZSM-5 and small pore silicoaluminophosphate (SAPO- $n$ ) molecular sieves have been examined as potential catalysts for these reactions [1-3]. Silicoaluminophosphate SAPO-34 with the topological 
structure of CHA has widely been studied in the MTO and DTO reactions. SAPO-18 molecular sieves with the topological structure of AEI also displays high selectivity toward light olefins as well as SAPO-34 [3, 4]. The pore opening of both SAPO-34 and SAPO-18 is composed of 8-membered oxygen rings and their structures are closely related. In comparison with ZSM-5, SAPO-18 and SAPO-34 show a high selectivity toward light olefins, but these catalysts are rapidly deactivated by a coke deposition, which completely blocks internal channels of the SAPO crystals.

Various methods have been suggested to improve a catalyst lifetime of small pore zeotype molecular sieve catalysts, such as the addition of water vapor in feed stream [5], the reduction of crystal size [6-10], the introduction of mesopore [11-14] and the control of acidity (acid strength and acid site density) [15-19]. Yuen et al. performed comparative tests of catalysts with CHA topology with varying acid strength (SAPO-34 and aluminosilicate SSZ-13, respectively) and acid site density ( $\mathrm{Si}$ content in SAPO-34 and Al content in SSZ-13, respectively) [15]. They reported that an intermediate acid site density is advantageous for the stability of SSZ-13 catalysts. In comparison, SAPO-34 with the same topology but lower acid strength was far more stable than SSZ-13 with similar acid site density. Bleken et al. also compared the stability of SAPO-34 and SSZ-13 [16]. They synthesized catalysts with similar acid site density and crystal size to eliminate the influence of these parameters on the catalytic properties of the materials, and elucidated the role of acid strength alone. They observed that SSZ-13 deactivated faster than SAPO-34 at the higher temperatures, while SSZ-13 displayed a higher conversion capacity at the lower temperatures.

These reports suggest that acidity of small pore zeotype molecular sieve catalysts is a very important factor in influencing the activity and lifetime on the MTO and DTO reactions. So far, various methods have been suggested to control acidity of zeolite and zeotype catalysts, such as ion exchange [17], and incorporation of metal into zeolite framework [18-20]. On the other hand, Si sources also affected the acidity of SAPO- $n$ catalyst $[13,14]$. Sun et al. synthesized mesoporous SAPO-34 molecular sieves using a quaternary ammonium-type organosilane surfactant as the mesoporogen and a part of the silica source. Compared to normal SAPO-34, the Brønsted acid strength of the mesoporous SAPO-34 was decreased [13]. Similar results were also reported by Wang et al. [14]. In the case of SAPO- $n$ molecular sieves, acidity depends on the microstructures of $\mathrm{Si}$ atom in the SAPO- $n$ framework [21, 22]. Using an organosilane as a part of Si source, microstructures of $\mathrm{Si}$ atom in the mesoporous SAPO-34 framework were changed, leading to the change of their acidity.

In contrast to these reports, in this study, SAPO-18 molecular sieve was synthesized by hydrothermal synthesis (HT) and dry gel conversion (DGC) method using an organosilane, methyltriethoxysilane (MTES) as the only Si source. Significant changes of microstructures of Si atoms can be expected, which may affect the acidity of SAPO-18 catalyst. In this paper, the effect of crystallization method on the synthesis of SAPO-18 molecular sieves using MTES was discussed. And their physicochemical and catalytic properties on DTO reactions were compared to those of conventional SAPO-18 molecular sieves. 


\section{Experimental}

\subsection{Catalysts preparation}

SAPO-18 molecular sieve was synthesized using an organosilane as a Si source by the HT and DGC method. Aluminum isopropoxide, $85 \mathrm{wt} \%$ phosphoric acid (both from Wako Pure Chemical Industries) and methyltriethoxysilane (MTES, Shin-Etsu Chemical) were used as sources of Al, P and $\mathrm{Si}$, respectively. A $35 \mathrm{wt} \%$ aqueous solution of tetraethylammonium hydroxide (TEAOH, Aldrich) was used as a structure direct agent (SDA). The molar ratio of precursor solution was 1.0 $\mathrm{Al}_{2} \mathrm{O}_{3}: 1.0 \mathrm{P}_{2} \mathrm{O}_{5}: x \mathrm{Si}: 2.0 \mathrm{TEAOH}: 50 \mathrm{H}_{2} \mathrm{O}(x=0.3$ or 0.6$)$. The precursor solution was stirred for $2 \mathrm{~h}$ at $60^{\circ} \mathrm{C}$. For the DGC method, the precursor solution was dried at $90^{\circ} \mathrm{C}$ for $12 \mathrm{~h}$. The obtained dry gel was placed in a Teflon-lined stainless steel vessel and a small amount of water, a source of steam, was separately added to the vessel. Crystallization was conducted at $180^{\circ} \mathrm{C}$ for 120 h. For the HT method, crystallization was carried out in a closed vessel at $180^{\circ} \mathrm{C}$ for $48 \mathrm{~h}$ with rotation. The products were rinsed with deionized water and finally calcined at $550^{\circ} \mathrm{C}$ for $6 \mathrm{~h}$. Here after, the obtained SAPO-18 sample is written as "crystallization method-Me-SAPO-18( $x)^{\text {", for }}$ example "DGC-Me-SAPO-18(0.6)".

In comparison, SAPO-18 crystals were also hydrothermally synthesized using colloidal silica (Aldrich) as a Si source. Aluminum hydroxide (Wako Pure Chemical Industries), phosphoric acid and N,N-diisopropylethylamine (DIPEA) (Tokyo Chemical Industries) were used as sources of aluminum, phosphorous and SDA, respectively. The molar composition of precursor solution was $1.0 \mathrm{Al}_{2} \mathrm{O}_{3}: 1.0 \mathrm{P}_{2} \mathrm{O}_{5}: x \mathrm{Si}: 2.0$ DIPEA : $50 \mathrm{H}_{2} \mathrm{O}(x=0.07,0.15$ and 0.3$)$. Crystallization was carried out in a closed vessel at $180^{\circ} \mathrm{C}$ for $48 \mathrm{~h}$ with rotation. Here after, the obtained SAPO-18 sample is written as "HT-SAPO-18(x)".

\subsection{Catalyst Characterization}

The products were identified from their X-ray diffraction (XRD) patterns recorded on the Philips $\mathrm{X}$ 'Pert-MPD diffractometer using $\mathrm{Cu}-\mathrm{K} \alpha$ radiation. Chemical compositions of $\mathrm{Al}, \mathrm{P}$ and $\mathrm{Si}$ of the products were measured by inductively coupled plasma (ICP; Perkin Elmer, OPTIMA). The morphology of the products was observed by TEM (Hitachi H-8000). The JEOL ECA-600 spectrometer was used for ${ }^{29} \mathrm{Si}$ MAS NMR. The porosity of the products was characterized by the $\mathrm{N}_{2}$ adsorption measurement at $-196^{\circ} \mathrm{C}$ using the BELSORP-max (MicrotracBEL). The acidic properties of the samples were analyzed by $\mathrm{NH}_{3}$-TPD measurement using the MicrotracBEL BELCAT II as following procedures. About $0.05 \mathrm{~g}$ of sample was pretreated under $\mathrm{He}$ flow at $500^{\circ} \mathrm{C}$ for $1 \mathrm{~h}$ and was subsequently cooled to the adsorption temperature of $100^{\circ} \mathrm{C}$. A gas mixture of $\mathrm{NH}_{3}$ and $\mathrm{He}\left(1 \mathrm{vol} \%\right.$ of $\mathrm{NH}_{3}$ ) was introduced into the sample cell for $1 \mathrm{~h}$. Subsequently, He (30 $\mathrm{cm}^{3}(\mathrm{STP}) / \mathrm{min}$ ) was passed through the sample to remove weakly adsorbed $\mathrm{NH}_{3}$ for $1 \mathrm{~h}$. Then, the sample was heated up to $600^{\circ} \mathrm{C}$ at the rate of $10^{\circ} \mathrm{C} / \mathrm{min}$. Desorbed $\mathrm{NH}_{3}$ signal was recorded on a quadrupole mass spectrometer (MicrotracBEL, BELMass). 


\subsection{Catalysis Testing}

DTO reactions over SAPO-18 catalysts were performed by using a fixed-bed reactor made of quartz glass (i.d. $4 \mathrm{~mm}$ ) with a continuous-flow system under atmospheric pressure. The feed rate of deimethylether (DME) was $1.88 \mathrm{mmol} / \mathrm{h}$, and reaction temperature was $400^{\circ} \mathrm{C}$. The amount of SAPO-18 catalyst was about $0.05 \mathrm{~g}$. The $W_{\text {cat }} / F_{\text {DME }}$ [mass of the catalyst $(\mathrm{g})$ divided by the feed rate of DME (mol/h)] was $26.7 \mathrm{~g} \mathrm{~h} / \mathrm{mol}$, and the partial pressure of DME was $9.21 \mathrm{kPa}$, respectively. The product stream was analyzed with the Shimazu GC-14B gas chromatograph equipped with a flame ionization detector using GS- $\mathrm{Al}_{2} \mathrm{O}_{3}$ PLOT column (J\&W scientific).

The amount of coke deposition was measured by thermogravimetry using the Shimadzu DTG-60 in a static air atmosphere with an increase of temperature at the rate of $5^{\circ} \mathrm{C} / \mathrm{min}$. The coke deposition was calculated from the mass ratio of $300^{\circ} \mathrm{C}$ to $600^{\circ} \mathrm{C}$.

\section{Results and discussions}

The XRD patterns of calcined samples are shown in Fig. 1 and S1. The XRD patterns show typical diffraction peaks of the AEI structure, proving that a pure phase of SAPO-18 was obtained from each solution by the HT and DGC method. The Si molar ratios of the products were compared in Table 1. The Si molar ratios of Me-SAPO-18(0.3) samples were smaller than that of HT-SAPO-18(0.3), although the Si molar ratios of precursor solutions for those samples were equal. This results indicate that organosilane, MTES, was difficult to incorporate to the SAPO-18 framework as a Si source. Additionally, comparison of Me-SAPO-18(0.3) samples suggests that SAPO-18 molecular sieves with high Si content could be synthesized by the DGC method, compared to the HT method. Fig. S2 shows ${ }^{29}$ Si MAS NMR spectrum of DGC-Me-SAPO-18(0.6) before calcination. The spectrum has two peaks at -57 and $-90 \mathrm{ppm}$, which were assigned to T-type of $\mathrm{Si}$ species bonded to carbon atoms, $\mathrm{SiR}(\mathrm{OH})_{\mathrm{n}}(\mathrm{OAl})_{3-\mathrm{n}}\left(\mathrm{T}^{\mathrm{n}}\right.$ type $)$ and $\mathbf{S i}(\mathrm{OH})_{n}(\mathrm{OAl})_{4-\mathrm{n}}\left(\mathrm{Q}^{\mathrm{n}}\right.$ type), respectively [23, 25]. These data confirm that organosilane, MTES, was incorporated to the SAPO-18 framework as a Si source. The ${ }^{29} \mathrm{Si}$ MAS NMR data indicated that Si-C bond of MTES was partially cleaved during the crystallization process and resultant $\mathrm{Si}\left(\mathrm{OC}_{2} \mathrm{H}_{5}\right)_{3} \mathrm{OH}$ species was preferentially incorporated to the SAPO-18 framework. In the case of the DGC method, crystallization is conducted in a high concentrated acid solution, leading to acceleration of cleavage of the $\mathrm{Si}-\mathrm{C}$ bond of MTES. Thus, decreased $\mathrm{Si}$ contents would be observed for the Me-SAPO-18(0.30) samples.

TEM images of SAPO-18 molecular sieves were shown in Fig. 2. The HT-SAPO-18(0.15) shows a cuboid morphology which is a typical AEI structure. The crystal size is smaller than $300 \mathrm{~nm}$. On the other hand, Me-SAPO-18 samples synthesized by both the HT and the DGC method were a flake-like crystal. The HR-TEM observation from the cross-sectional views of the DGC-Me-SAPO-18(0.3) revealed that the thickness in the $c$-direction of the nanosheets is about 30 $\mathrm{nm}$ and, lattice fringes were also clearly observed (Fig. S3). These results indicate that SAPO-18 molecular sieve with high crystallinity was successfully synthesized using only MTES as a Si 
source. TEM observation revealed that the crystallization method did not affect the morphology of Me-SAPO-18 samples. When colloidal silica and TEAOH were used as Si source and SDA, SAPO-34 molecular sieves with the topological structure of CHA were obtained by both DGC and HT methods. Thus, morphological change from a cuboid crystal to a flake-like crystal must be due to the effect of SDA and Si source.

The $\mathrm{N}_{2}$ adsorption isotherms for HT-SAPO-18(0.15) and DGC-Me-SAPO-18(0.6) are presented in Fig. 3. Both samples showed the type-I isotherms. Compared with HT-SAPO-18(0.15), micropore volume of DGC-Me-SAPO-18(0.6) was slightly increased. The flake-like SAPO-18 with high crystallinity was successfully obtained using only MTES as a Si source. This result agrees with the TEM observation. Possible reason for the increase of micropore volume for DGC-Me-SAPO-18(0.6) was due to the formation of microstructural defects by thermal decomposition of methyl groups.

The distributions of acid strength measured using $\mathrm{NH}_{3}$-TPD are shown in Fig. 4. Each SAPO-18 molecular sieve showed two desorption peaks at $200^{\circ} \mathrm{C}$ (L-peak) and at $300-400^{\circ} \mathrm{C}$ (H-peak). The L-peak is due to the Lewis acid sites originated from surface $\mathrm{POH}, \mathrm{SiOH}$ or $\mathrm{AlOH}$ groups, and the $\mathrm{H}$-peak is due to the Brønsted acid sites in the form of bridging hydroxyl groups [23-25]. It is well known that Brønsted acid sites contribute to the formation of hydrocarbons from DME. Lewis acid sites show an activity for dehydration of methanol, but do not contribute to synthesis of hydrocarbon from DME. The amount of Brønsted acid sites, which is equal to peak area of H-peak, for HT-SAPO-18 samples decreased with Si content, although the peak position of H-peak for HT-SAPO-18 samples was not changed. This result indicated that Brønsted acid strength for each HT-SAPO-18 samples was the same, but the amount of acid sites was different. On the other hand, the peak positions of H-peaks for Me-SAPO-18 samples moved to lower temperature, indicating that Brønsted acid strength of Me-SAPO-18 samples was lower than that of HT-SAPO-18 samples. The weakened Brønsted acid for Me-SAPO-18 would be due to the formation of microstructural defects by thermal decomposition of methyl groups. Another possible reason was the difference of incorporated Si sites. In the case of SAPO-34 molecular sieves, two distinct Brønsted acid sites corresponding to protons residing on inequivalent oxygen atoms have been revealed on the basis of FT-IR experiments [21]. Similarly to SAPO-34 molecular sieve, SAPO-18 would also possess Brønsted acid sites with different strength. Due to lower reactivity of MTES, Si atoms seem to be incorporated to the particular site with lower acidity.

The catalytic properties on the DTO reaction were compared between HT-SAPO-18(0.15) and DGC-Me-SAPO-18(0.6). Because the amount of Brønsted acid of both SAPO-18 samples was similar (HT-SAPO-18(0.15); $1.4 \mathrm{mmol} / \mathrm{g}$, DGC-Me-SAPO-18(0.6); $1.2 \mathrm{mmol} / \mathrm{g}$ ). The time courses of DME conversion and hydrocarbons yield are plotted in Fig. 5. The DME conversions and yields of light olefins (ethylene + propylene + butene) over DGC-Me-SAPO-18(0.6) were decreased at a slower rate than those of HT-SAPO-18(0.15), indicating that DGC-Me-SAPO-18(0.6) exhibited a prolonged catalytic lifetime compared with HT-SAPO-18(0.15). The amount of produced light olefins over DGC-Me-SAPO-18(0.6) was more than 3 times as much as HT-SAPO-18(0.15). Additionally, the yield of light alkanes $\left(\mathrm{C}_{1}-\mathrm{C}_{4}\right)$ over DGC-Me-SAPO-18(0.6) was decreased 
compared to that over DGC-Me-SAPO-18(0.6) (Fig. 5 and Fig. S4). This result indicates that decreased Brønsted acidic strength suppressed the transformation of olefins to paraffins. From the $\mathrm{NH}_{3}$-TPD analysis, a significant difference in the amount of Brønsted acid between two catalysts was not observed. Thus, prominent difference in the catalytic performance of DGC-Me-SAPO-18(0.6) can be explained by the difference in their acidity. The coke deposition rate on both catalysts were shown in Fig. 6. The coke was deposited on DGC-Me-SAPO-18(0.6) at a slower rate than HT-SAPO-18(0.15). Another possible reason for the excellent lifetime on DGC-Me-SAPO-18(0.6) was its reduced diffusion path in the crystals. Due to its thin thickness, the olefinic products like ethylene and propylene can easily diffuse out of the crystal before their further reactions including coke formation. The amount of coke on deactivated DGC-Me-SAPO-18(0.6) was larger than HT-SAPO-18(0.15). The decreased acidic strength and flake-like morphology of DGC-Me-SAPO-18(0.6) could retard the coke formation, and the reactants and produced hydrocarbon could reach the centers of the catalyst particles.

\section{Conclusions}

SAPO-18 molecular sieve was successfully obtained by the HT and DGC method using MTES as a $\mathrm{Si}$ source (Me-SAPO-18). TEM observation and $\mathrm{N}_{2}$ adsorption analysis revealed that the Me-SAPO-18 with high crystallinity was synthesized by the DGC method (DGC-Me-SAPO-18). The use of MTES as a $\mathrm{Si}$ source affected the acidity of DGC-Me-SAPO-18. Compared to conventional SAPO-18, Brønsted acid strength for DGC-Me-SAPO-18 was decreased. The DGC-Me-SAPO-18 catalyst exhibited a prolonged catalytic lifetime compared to conventional SAPO-18. Its weak acidity and flake-like morphology could suppressed the coke formation, and thus the reactants and produced hydrocarbon could reach the centers of the catalyst particles, leading to the increase of catalyst effectiveness factor.

\section{Acknowledgement}

This work was financially supported in part by CREST program from Japan Science and Technology Agency (JST). Authors are grateful to Mr. Shigeru Tamiya (Center for Scientific Instrument Renovation and Manufacturing Support, Osaka University) for ICP analysis. The TEM measurements were carried out by using a facility in the Research Center for Ultrahigh Voltage Electron Microscopy, Osaka University. 


\section{References}

[1] W.W. Kaeding, S.A. Butter, J. Catal. 61 (1980) 155.

[2] J. Liang, H. Li, S. Zhao, W. Guo, R. Wang and M. Ying, Appl. Catal. 64 (1990) 31.

[3] R. Wendelbo, D. Akporiaye, A. Andersen, I.M. Dahl, H.B. Mostad, Appl. Catal. A: Gen. 142 (1996) L197.

[4] A.T. Aguayo, A.G. Gayubo, R. Vivanco, M. Olazar, J. Bilbao, Appl. Catal. A: Gen. 283 (2005) 197.

[5] A.J. Marchi, G.F. Froment, Appl. Catal. 71 (1991) 139.

[6] S. Wilson, P. Barger, Micropor. Mesopor. Mater. 29 (1999) 117.

[7] D. Chen, K. Moljord, T. Fuglerud and A. Holmen, Micropor. Mesopor. Mater. 29 (1999) 191.

[8] Y.J Lee, S.C. Baek, K.W. Jun, Appl. Catal. A: Gen. 329 (2007) 130.

[9] N. Nishiyama, M. Kawaguchi, Y. Hirota, V.D. Van, Y. Egashira, K. Ueyama, Appl. Catal. A: Gen. 362 (2009) 193.

[10] Y. Hirota, K. Murata, M. Miyamoto, Y. Egashira, N. Nishiyama, Catal. Lett. 140 (2010) 22.

[11] Y. Liu, L. Wang, J. Zhang, L. Chen, H. Xu, Micropor. Mesopor. Mater. 145 (2011) 150.

[12] F. Schmidt, S. Paasch, E. Brunner, S. Kaskel, Micropor. Mesopor. Mater. 164 (2012) 214.

[13] Q. Sun, N. Wang, D. Xi, M. Yang, J. Yu, Chem. Commun. 50 (2014) 6502.

[14] C. Wang, M. Yang, P. Tian, S. Xu, Y. Yang, D. Wang, Y. Yuan, Z. Liu, J. Mater. Chem. A 3 (2015) 5608.

[15] L.T. Yuen, S.I. Zones, T.V. Harris, E.J. Gallegos, A. Auroux, Micropor. Mater. 2 (1994) 105.

[16] F. Bleken, M. Bjørgen, L. Palumbo, S. Bordiga, S. Svelle, K.P. Lillerud, U. Olsbye, Top. Catalysis 52 (2009) 218.

[17] M.M. Abdillahi, U.A. El-Nafaty, A.M. Al-Jarallah, Appl. Catal. A: Gen. 91 (1992) 1.

[18] T Inui, M. Kang, Appl. Catal. A: Gen. 164 (1997) 211.

[19] L. Regli, S. Bordiga, C. Lamberti, K.P. Lillerud, S.I. Zones, A. Zecchina, J. Phys. Chem. C, 111 (2007) 2292.

[20] M. Salmasi, S. Fatemi, A.T. Najafabadi, J. Ind. Eng. Chem. 17 (2011) 755.

[21] L. Smith, A.K. Cheetham, L. Marchese, J.M. Thomas, P.A. Wright, J. Chen, Catal. Lett. 41 (1996) 13.

[22] W. Shen, Z. Li, Y. Wei, P. Tian, F. Deng, X. Han, X. Bao, Micropor. Mesopor. Mater. 158 (2012) 19.

[23] S. Ashtekar, S.V.V. Chilukuri, D.K. Chakrabary, J. Phys. Chem. 98 (1994) 4878.

[24] E. Dumitriu, A. Azzouz, V. Hulea, D. Lutic, H. Kessler, Micropor. Mesopor. Mater. 10 (1997) 11.

[25] M. Kang, J. Mol. Catal. A Chem. 160 (2000) 437. 


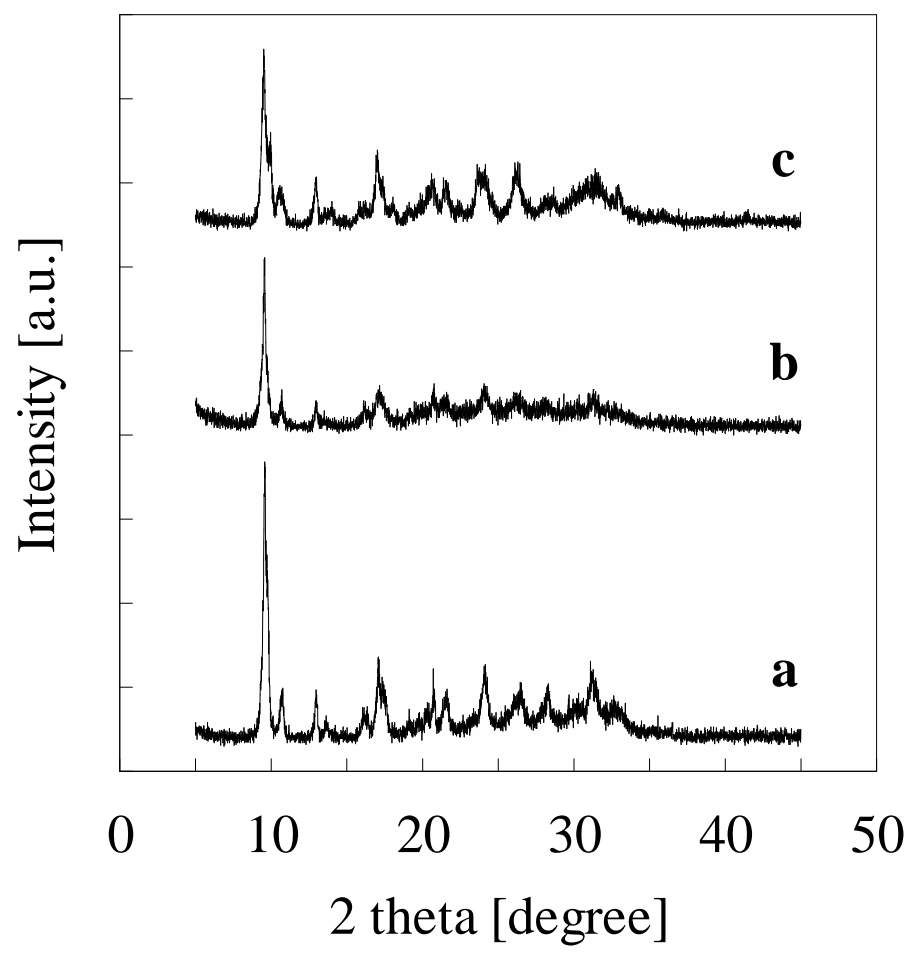

Fig. 1 XRD patterns of (a) DGC-Me-SAPO-18(0.6), (b) HT-Me-SAPO-18(0.3) and (c) HT-SAPO-18(0.15). 
Table 1 Si ratios of SAPO-18 samples.

\begin{tabular}{lc}
\hline \multicolumn{1}{c}{ Sample } & Molar ratio of Si/(Al+P+Si) [-] \\
\hline HT-SAPO-18(0.30) & 0.074 \\
HT-SAPO-18(0.15) & 0.060 \\
HT-SAPO-18(0.07) & 0.024 \\
DGC-Me-SAPO-18(0.6) & 0.037 \\
DGC-Me-SAPO-18(0.3) & 0.016 \\
HT-Me-SAPO-18(0.3) & 0.012 \\
\hline
\end{tabular}



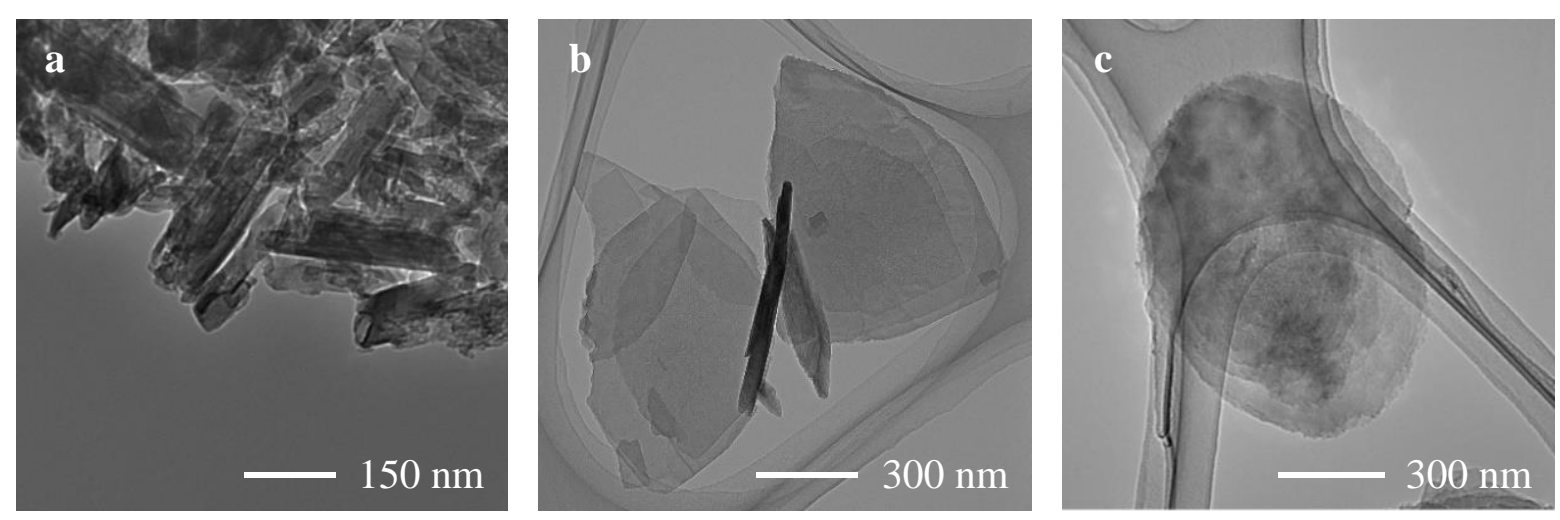

Fig. 2 TEM image of (a) HT-SAPO-18, (b) HT-Me-SAPO-18(0.3) and (c) DGC-Me-SAPO-18(0.6). 


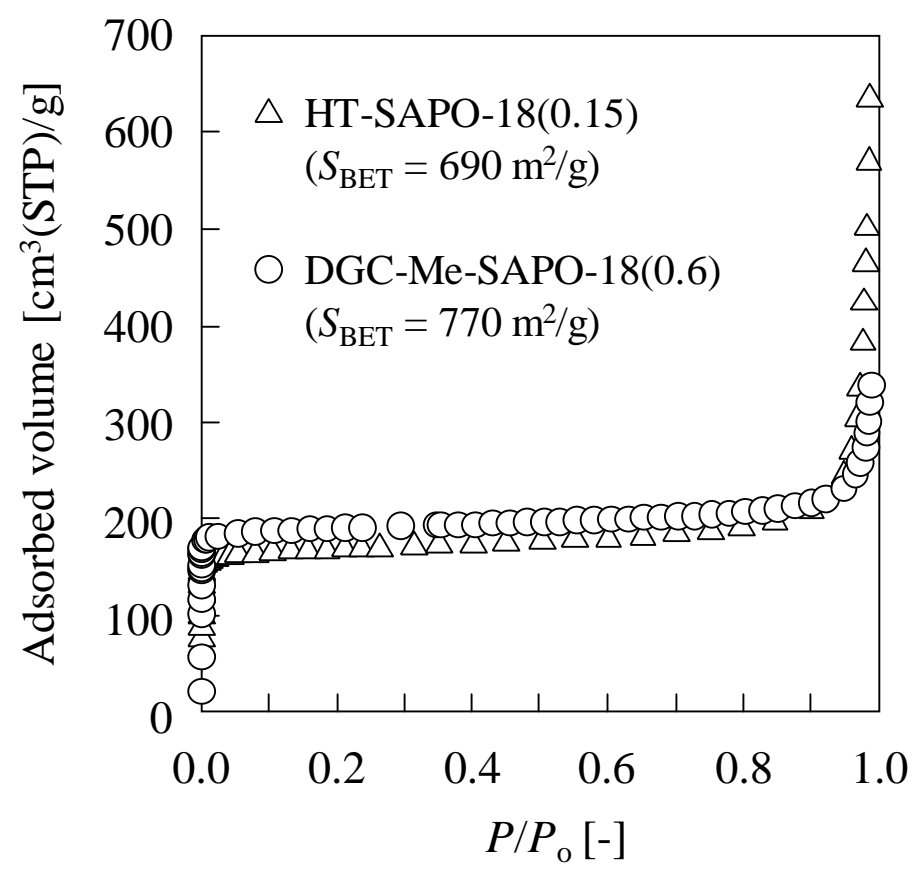

Fig. $3 \mathrm{~N}_{2}$ adsorption isotherms of SAPO-18 samples. 


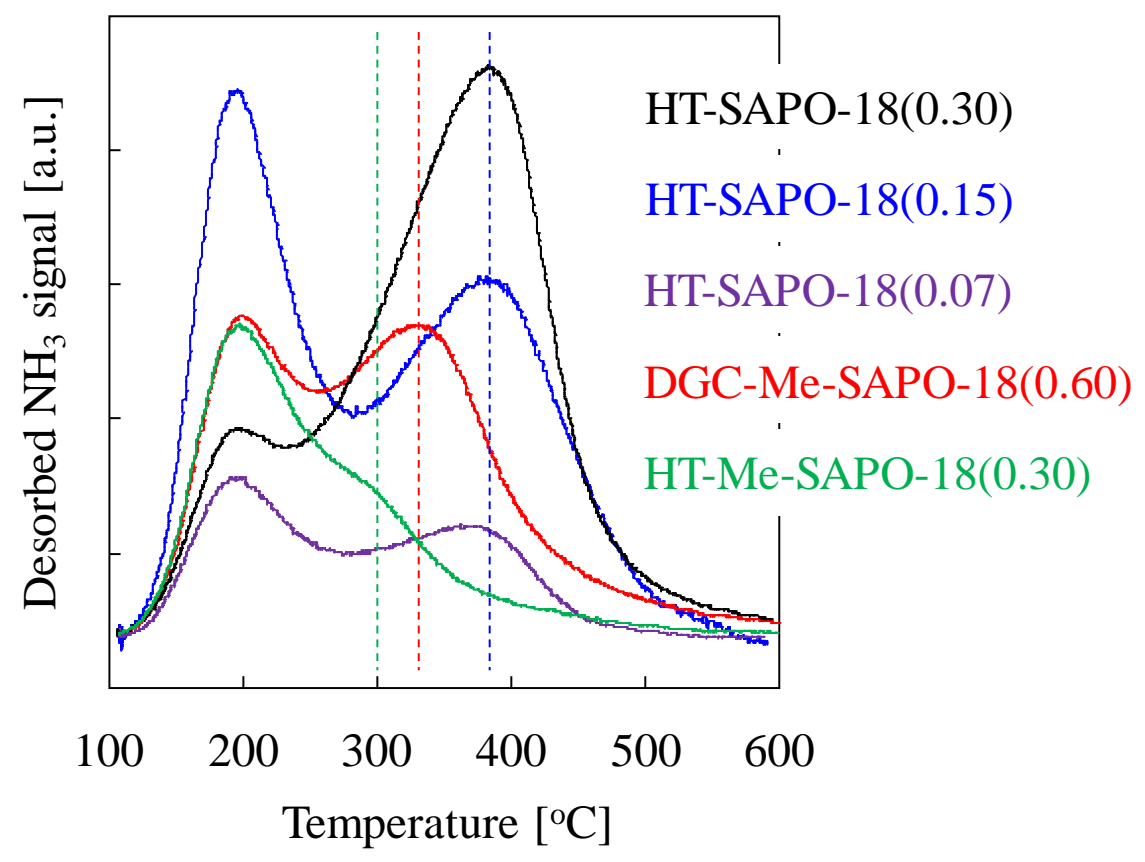

Fig. $4 \mathrm{NH}_{3}$-TPD curves for SAPO-18 samples. 

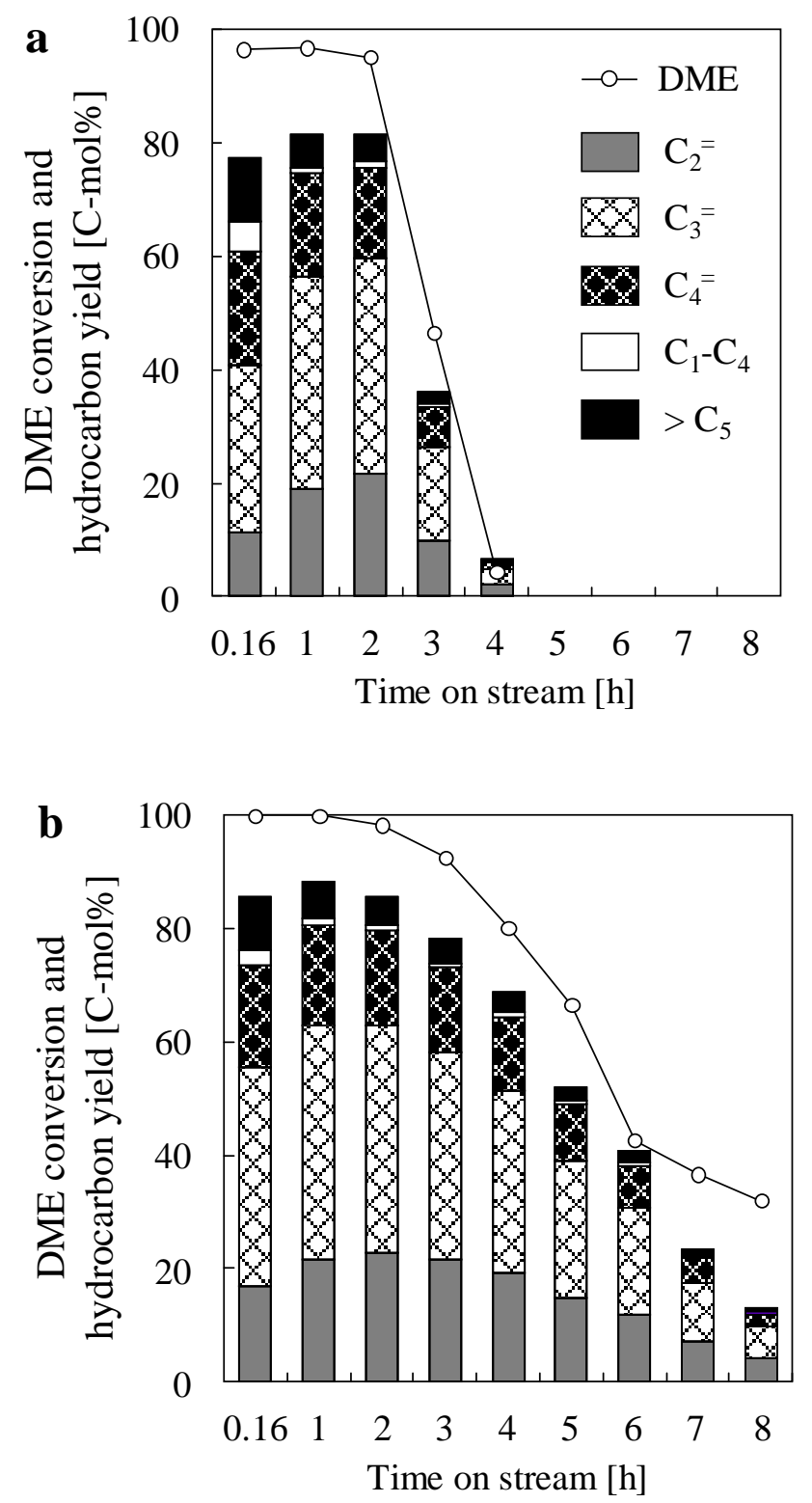

Fig. 5 Time sources of DME conversion and hydrocarbon yield over (a) HT-SAPO-18(0.15) and (b) DGC-Me-SAPO-18(0.6). 


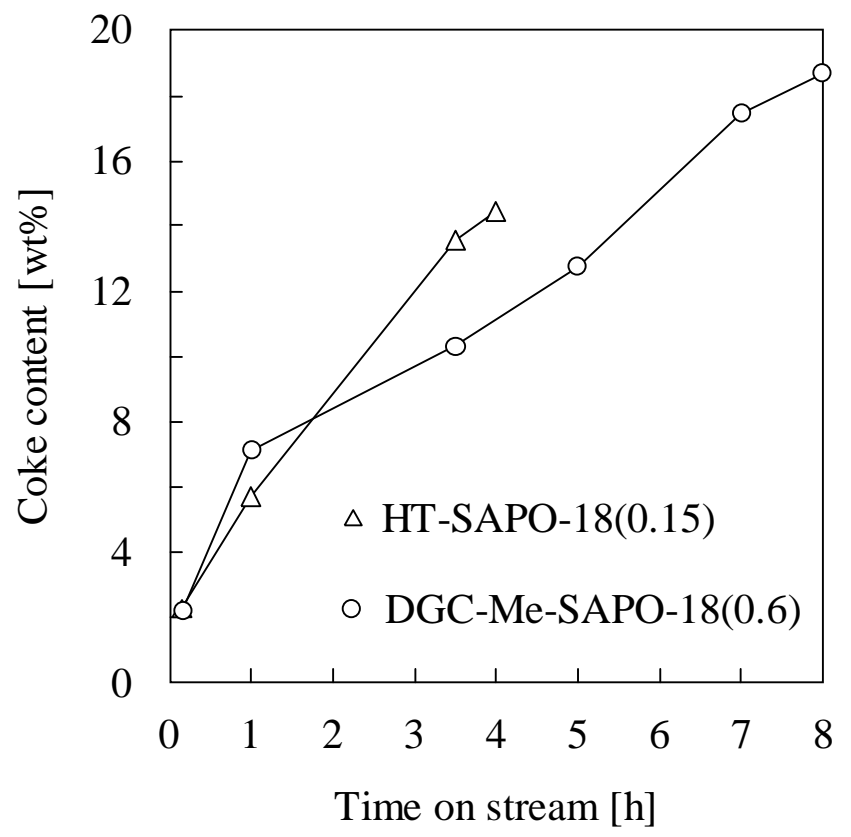

Fig. 6 The coke deposition rate on the SAPO-18 samples. 
Conventional SAPO-18

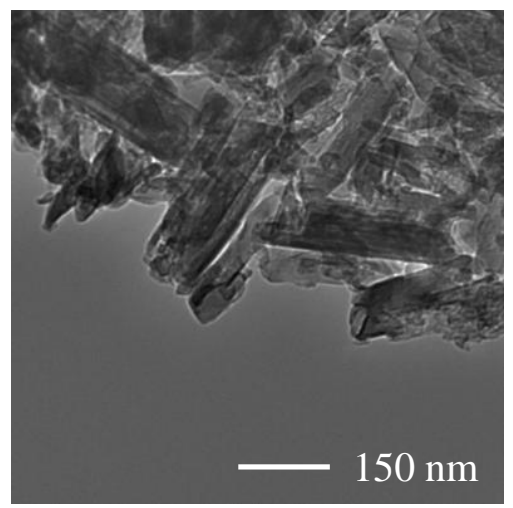

Flake-like SAPO-18 with low acidic strength

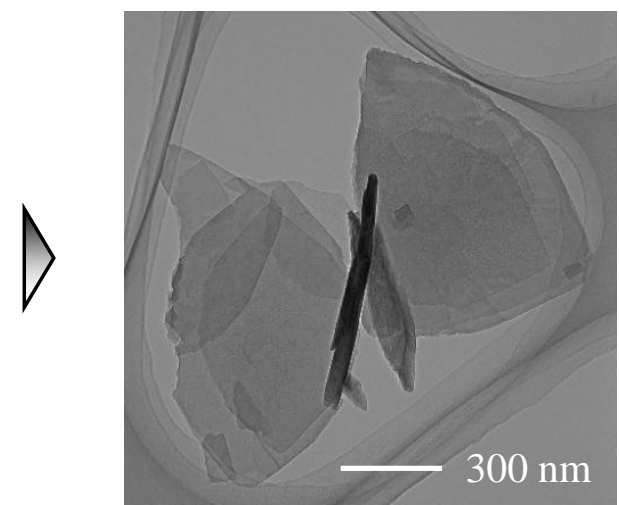

